\title{
Classical Hodgkin Lymphoma Presenting as a Sigmoid Mass
}

\author{
Heather Caulkins ${ }^{\mathrm{a}}$, Alyaa Irhayyima ${ }^{\mathrm{a}}$, Lijun Yang ${ }^{\mathrm{a}}$, Xiuli Liu ${ }^{\mathrm{a}}$,
}

\begin{abstract}
Primary classical Hodgkin lymphoma (CHL) in the colon is exceedingly rare and shares many histologic features with other lymphoproliferative disorders in the gastrointestinal tract. Here we report a case of CHL forming a sigmoid mass. An elderly man with a past medical history of mantle cell lymphoma presented with constipation. Imaging revealed an ulcerated, circumferential mass in the sigmoid colon. Endoscopic biopsy of the mass showed ulcerated colonic mucosa with an underlying diffuse mixed inflammatory infiltrate admixed with Hodgkin and Reed-Sternberg cells. Immunohistochemistry was performed to characterize these cells. They were weakly positive for Pax-5, strongly positive for CD30, variably positive for CD15, and negative for CD45, CD20, CD3, and SOX-11. In situ hybridization was positive for Epstein-Barr virus (EBV) and negative for cytomegalovirus or herpes simplex virus. This immunophenotype is diagnostic for $\mathrm{CHL}$ in the clinical context of a large mass. It is not possible in this case to determine whether this is de novo CHL or progression from a precursor lesion like EBV-positive mucocutaneous ulcer. Since diagnosis, this patient underwent colectomy followed by chemotherapy and has remained in complete remission.
\end{abstract}

Keywords: Hodgkin lymphoma; EBV; Gastrointestinal lymphoma; EBV-positive mucocutaneous ulcer

\section{Introduction}

The gastrointestinal (GI) tract is a relatively uncommon location for primary extra-nodal lymphomas. Upon endoscopic biopsy, entities such as diffuse large B-cell lymphoma (DLBCL), follicular lymphoma, and mucosa-associated lymphoid tissue (MALT) lymphoma are the most common diagnoses [1]. Primary classical Hodgkin lymphoma (CHL) involving the GI

Manuscript submitted May 6, 2021, accepted June 3, 2021

Published online June 19, 2021

aDepartment of Pathology, Immunology and Laboratory Medicine, University of Florida, Gainesville, FL, USA

${ }^{b}$ Corresponding Author: Xiuli Liu, Department of Pathology, Immunology and Laboratory Medicine, College of Medicine, University of Florida, 1600 SW Archer Road, Gainesville, FL 32610, USA. Email: xiuliliu@ufl.edu

doi: https://doi.org/10.14740/gr1409 tract is exceedingly rare [2]. In previous cases where CHL is identified within the GI tract, it has been linked to clinical histories such as non-Hodgkin lymphoma, inflammatory bowel disease, and immunosuppression [3, 4]. A newly described entity, Epstein-Barr virus-positive mucocutaneous ulcer (EBVMCU), shows features which overlap with CHL [5, 6]. Differentiation of these two diagnoses is critical for guiding appropriate treatment decisions as EBVMCU may respond to reduction in immunosuppressant while CHL may need chemotherapy. Here we report a case of primary CHL presenting as a sigmoid mass.

\section{Case Report}

\section{Investigations}

The case was an 84-year-old man with a past history of mantle cell lymphoma. Four years ago, he presented to an outside facility with weight loss, fatigue, and a left axillary mass. The $5-\mathrm{cm}$ axillary mass was excised and diagnosed as mantle cell lymphoma, stage 4B (an advanced stage with mass formation and non-nodal involvement). Fluorescent in situ hybridization (FISH) detected an IGH/CCND1 fusion rearrangement, supporting the diagnosis of mantle cell lymphoma. The patient was treated for his mantle cell lymphoma, but the exact treatment and remission status is unknown.

More recently, he presented to an outside hospital with worsening constipation. Imaging by positron emission tomography/computed tomography (PET/CT) showed increased radiotracer uptake in his GI tract, which was greatest in the distal colon without significant nodal or splenic uptake. Subsequent colonoscopic evaluation revealed a $10-\mathrm{cm}$ in length circumferential, ulcerating and infiltrative mass in the sigmoid colon and no obstruction. This mass was biopsied, and the biopsy material was sent to our institution for diagnostic consultation.

\section{Diagnosis}

The biopsy fragments showed ulcerated colonic tissue. The mucosal and submucosal tissue was distorted by a diffuse infiltrate of mixed inflammation consisting of predominantly neutrophils with scattered eosinophils, lymphocytes, histiocytes, and plasma cells (Fig. 1a). Glandular dysplasia or carcinoma were not present within the biopsied material. Most of 

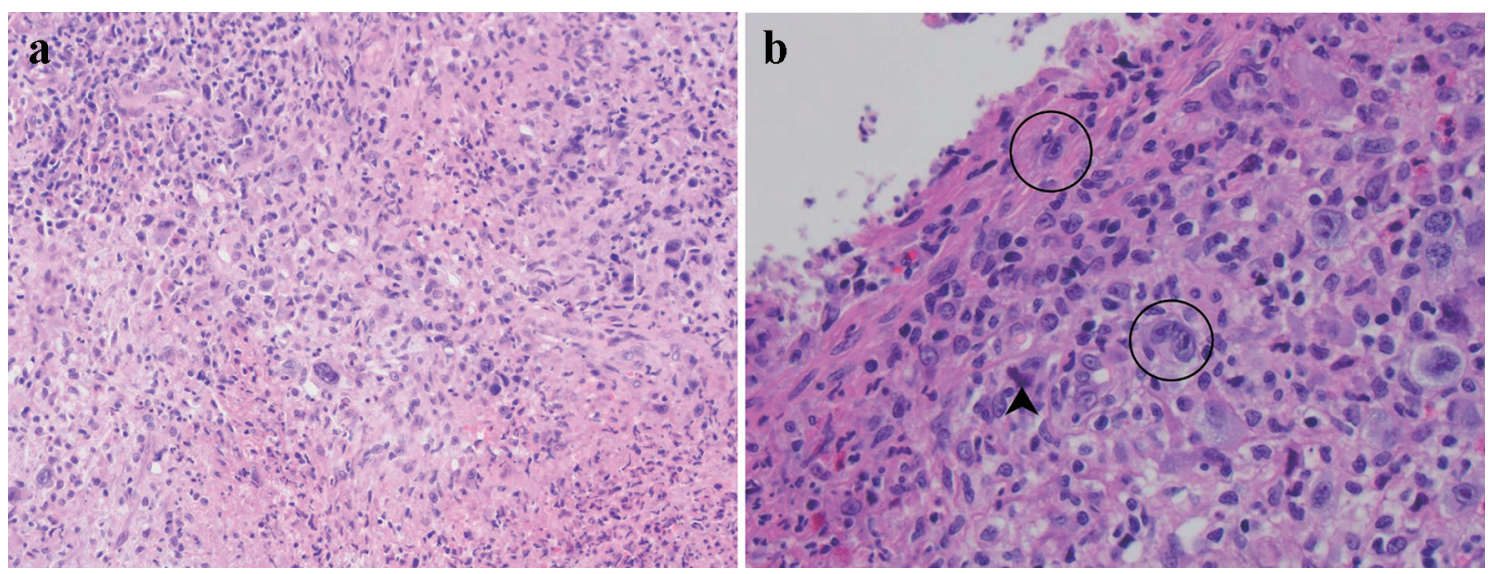

Figure 1. Histological evaluation of lesional tissue by hematoxylin and eosin (H\&E) stain: (a) the lesion consists primarily of a destructive infiltrate of large, atypical cells mixed in a background of neutrophils, small lymphocytes, and eosinophils $(\times 100$ magnification); (b) the large atypical cells resemble Hodgkin or Reed-Sternberg cells (circled) with 1 - 2 nuclei featuring vesicular chromatin and prominent inclusion-like nucleoli (× 400 magnification). Mummified cells are also depicted (arrowhead).

the small lymphocytes were $\mathrm{T}$ cells by immunohistochemistry. Also scattered throughout the lesion were large, atypical cells morphologically resembling Hodgkin or Reed-Sternberg cells. These atypical cells demonstrated abundant pale cytoplasm, fine chromatin, and a single inclusion-like prominent nucleolus in each nucleus (Fig. 1b). Occasionally, these atypical cells were bi- or multi-nucleated. Mitotic activity within the lesion was predominantly observed within these large cells. Also scattered within the inflammatory milieu was a second population of large, atypical cells which showed condensed cytoplasm and blurred nuclei (so-called "mummified cells").

Immunohistochemistry was performed to characterize the large, atypical neoplastic cells (Fig. 2). These cells were negative for CD45/leukocyte common antigen (Fig. 2a), CD20 (Fig. 2b), CD3 and other T-cell markers, SOX-11, Bcl-6, cyclin-D1, and ALK. They were weakly positive for Pax-5 (Fig. 2c), CD79a, Bcl-2, OCT-2, and MUM-1. They were positive for CD30 (Fig. 2d) and variably positive for CD15 (Fig. 2e). They did not express kappa and lambda surface or cytoplasmic light chains by chromogenic in situ hybridization (CISH). CISH revealed that these large atypical cells were positive for EBV (Fig. 2f) and negative for cytomegalovirus and herpes simplex virus. The overall immunophenotype and CISH EBV result supported an EBV-positive B-cell neoplasm with abundant reactive T cells, features of CHL. Due to the rarity of this entity, this case has been reviewed at an intradepartmental consensus conference, and a consensus diagnosis of CHL, mixed cellularity variant was made given the clinical presentation of large colonic mass and the above immunophenotype.

\section{Treatment and outcomes}

In this case, the patient developed life-threatening lower GI tract hemorrhage. He underwent colectomy for this deep and large ulcerating mass. He responded well to adjuvant chemotherapy, achieving complete remission 5 months after the initial diagnosis.

\section{Discussion}

The imaging and endoscopic findings of the sigmoid lesion in this case were highly concerning for a primary colonic malignancy, the top differential diagnosis being colonic adenocarcinoma. Prominent lymphoid response to colonic adenocarcinoma can occur, and when lymphoproliferation masks underlying carcinoma, the carcinomatous cells may be identified using cytokeratin and CDX2 immunostains [7]. However, the biopsy fragments in this case clearly showed a lymphoproliferative tumor based on the histomorphology and immunophenotype. The destructive lesion primarily involved the submucosa with limited extension into the mucosa. There was no evidence of chronic colitis, epithelial dysplasia, or carcinoma.

The biopsy showed a diffuse infiltrate of mixed inflammation consisting of predominantly neutrophils with scattered eosinophils, lymphocytes, histiocytes, and plasma cells. The scattered large atypical cells showed morphologic features compatible with Hodgkin/Reed-Sternberg cells or so-called mummified cells. Mitotic activity within the lesion was predominantly observed within these large cells rather than the accompanying inflammatory milieu. Immunohistochemical staining and CISH revealed that these large atypical cells were EBV-infected, CD30-positive and CD15 variably positive B lymphocytes lacking expression of CD45 and CD20. Most of the small lymphocytes were $T$ cells by immunohistochemical staining. These features are most consistent with CHL, mixed cellularity variant.

The most common non-Hodgkin B-cell lymphomas in the colon include DLBCL, MALT lymphoma, and follicular lymphoma [8]. While T-cell rich DLBCL can mimic CHL, the large, atypical lymphocytes in DLBCL express CD20 and strong Pax-5 [9], unlike this case. MALT lymphoma tends to form lymphoepithelial lesions rather than causing ulceration and destruction of the surrounding tissue, and the predominant cell type would be small CD20-positive B cells [10]. High-grade follicular lymphoma can present with a more diffuse infiltrative pattern; however, this would not be T-cell rich, and the lesional 

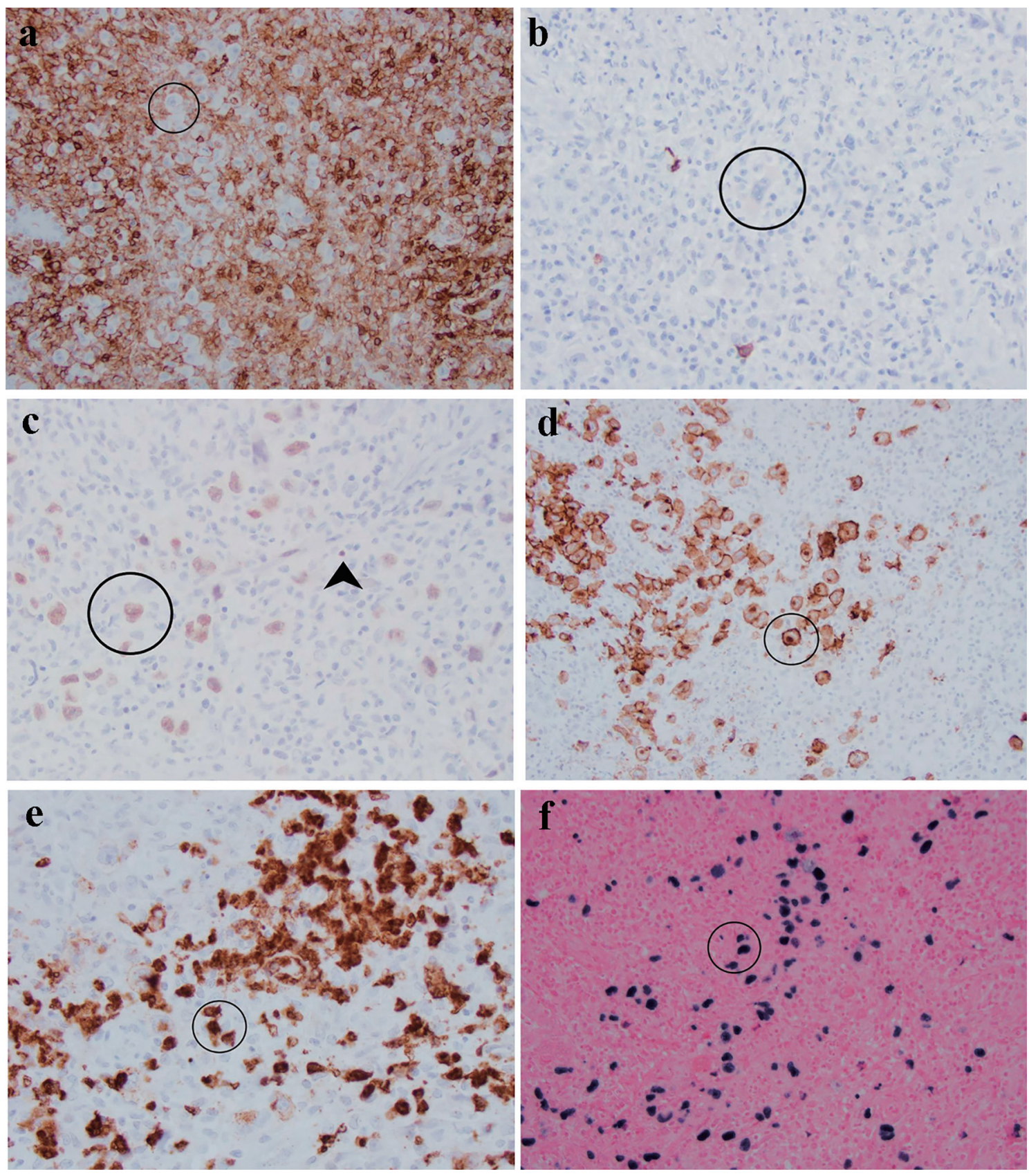

Figure 2. Characterization of atypical cells (circled) in this sigmoid mass by immunohistochemistry and in situ hybridization: (a) CD45 is negative in comparison with surrounding lymphocytes; (b) CD20; (c) Pax-5 is weak in Reed-Sternberg cells in comparison with strong staining in background B cells (arrowhead); (d) CD30 highlights membrane and Golgi bodies; (e) CD15 is positive in approximately $75 \%$ of Reed-Sternberg cells; (f) Epstein-Barr virus is positive in large cells rather than background small lymphocytes.

cells would be CD45- and CD20-positive and co-express Bcl-2 and Bcl-6 [11]. Given the morphologic and immunohistochemical patterns in this biopsy specimen, none of these common B-cell lymphomas are likely diagnoses over CHL.

Given the patient's prior history, another possibility was extranodal recurrence of mantle cell lymphoma. In particular, the large, atypical cells could represent the more aggressive pleomorphic variant. The large cells in pleomorphic mantle cell lymphoma should stain positively for CD20, CD5, and SOX-11 $[12,13]$, which was not seen in this case. While this lymphoma was not recurrence of mantle cell lymphoma, it is possible that immunosuppression from induction or maintenance treatment in this patient contributed to the pathogenesis of CHL.

Peripheral T-cell lymphomas (PTCLs) can also mimic CHL in the GI tract. In these instances, cells morphologically 
similar to Reed-Sternberg cells are present and share an identical immunophenotype as the neoplastic T cells. Additionally, CHL can arise within tissue involved by a T-cell lymphoma due to the immunosuppressive effects of the underlying PTCL [12]. In either setting, the background $T$ cells should aberrantly lose expression of T-cell markers such as CD3, CD4, CD7, and CD8, and may aberrantly express CD10 or Bcl-6. Complete differentiation from $\mathrm{CHL}$ requires molecular studies for T-cell clonality $[12,14,15]$. In our case, immunohistochemical stains showed no abnormal patterns among the background $\mathrm{T}$ cells, making underlying T-cell lymphoma unlikely.

Primary CHL in the colon is exceedingly rare and has been associated with immunosuppression and inflammatory bowel disease [4]. In most cases of CHL in the GI tract, colonic involvement represents metastasis from a nodal primary or Richter transformation from chronic lymphocytic leukemia/small lymphocytic lymphoma (CLL/SLL) [16, 17]. This patient's PET/CT scan did not show lymphadenopathy or foci of significant radiotracer uptake within lymph nodes or the spleen, making extranodal metastasis unlikely in this case. In Richter transformation, the Reed-Sternberg cells can be positive for EBV [18] and tend to be positive for MUM-1 [12]. Due to the immunohistochemical staining pattern and lack of areas morphologically resembling CLL/SLL in other fragments in the biopsy, primary CHL is more likely than transformation of a previous lymphoma. Histological review of the colectomy specimen would be helpful to completely rule out this possibility, but the surgery was performed at an outside hospital and the colectomy specimen was not available to us to review.

EBVMCU is a newly described entity presenting as a small ulcer rather than a large mass. EBVMCU is usually associated with immunosuppression or immune senescence. The lesions in EBVMCU are reported to be well circumscribed and superficial $[5,6,19]$. They can mimic CHL with ReedSternberg-like (RSL) cells admixed with infiltrating B lymphocytes. These RSL cells are positive for CD45, CD20 and EBV, and can also express CD15 and CD30 [5, 6, 19]. Most of these cases have an indolent clinical course and often resolve without treatment; however, complications such as luminal obstruction have been described [20]. Given the large size of the patient's mass lesion, EBVMCU is less likely than CHL in this case. Whether our current case represents a de novo CHL or progression from an EBVMCU is not clear. Recently, a case of colonic EBVMCU progressing to CHL, mixed cellularity variant in less than 2 years was reported in a patient with Crohn's disease [21]. It is possible that our case is immunodeficiency (immunosuppression or immune senescence)-related given the patient's age of 84 years and a clinical history of mantle cell lymphoma which has been treated.

In summary, when biopsy of a colonic mass shows atypical cells in a mixed inflammatory milieu, a diagnosis of CHL of the GI tract must be in the differential, although primary CHL of the GI tract is extremely rare. Immunohistochemistry using a panel of antibodies helps rule out non-Hodgkin lymphomas such as DLBCL, MALT lymphoma, follicular lymphoma, and PTCL. Primary CHL of the GI tract can only be established after GI tract (extra-nodal) involvement by nodal CHL and transformation into CHL from low-grade lymphomas are ruled out by clinicopathologic correlation. It is essential to distinguish
CHL from EBVMCU as treatments are different. CHL may need chemotherapy and in some cases surgery, while EBVM$\mathrm{CU}$ may respond to reduction in immunosuppression in cases secondary to immunosuppressant treatment.

\section{Acknowledgments}

None to declare.

\section{Financial Disclosure}

None to declare.

\section{Conflict of Interest}

None to declare.

\section{Informed Consent}

Per Institutional Review Board guidelines, informed consent is not required for this de-identified, single patient case report.

\section{Author Contributions}

Conceptualization: XL; investigation: HC, AI, LY, and XL; resources: $\mathrm{HC}$ and $\mathrm{XL}$; writing original draft preparation: $\mathrm{HC}$; writing review and editing: AI, LY, and XL; visualization: $\mathrm{HC}$; supervision: XL; project administration: XL. All authors have read and agreed to the published version of the manuscript.

\section{Data Availability}

The authors declare that data supporting the findings of this study are available within the article.

\section{References}

1. Peng JC, Zhong L, Ran ZH. Primary lymphomas in the gastrointestinal tract. J Dig Dis. 2015;16(4):169-176.

2. Devaney K, Jaffe ES. The surgical pathology of gastrointestinal Hodgkin's disease. Am J Clin Pathol. 1991;95(6):794-801.

3. Zarate-Osorno A, Medeiros LJ, Kingma DW, Longo DL, Jaffe ES. Hodgkin's disease following non-Hodgkin's lymphoma. A clinicopathologic and immunophenotypic study of nine cases. Am J Surg Pathol. 1993;17(2):123132.

4. Kumar S, Fend F, Quintanilla-Martinez L, Kingma DW, Sorbara L, Raffeld M, Banks PM, et al. Epstein-Barr virus-positive primary gastrointestinal Hodgkin's disease: association with inflammatory bowel disease and immu- 
nosuppression. Am J Surg Pathol. 2000;24(1):66-73.

5. Prieto-Torres L, Erana I, Gil-Redondo R, Gomez de la Riva I, Manso R, Pajares R, Cordoba R, et al. The spectrum of EBV-positive mucocutaneous ulcer: a study of 9 cases. Am J Surg Pathol. 2019;43(2):201-210.

6. Ikeda T, Gion Y, Sakamoto M, Tachibana T, Nishikori A, Nishimura MF, Yoshino T, et al. Clinicopathological analysis of 34 Japanese patients with EBV-positive mucocutaneous ulcer. Mod Pathol. 2020;33(12):2437-2448.

7. Werling RW, Yaziji H, Bacchi CE, Gown AM. CDX2, a highly sensitive and specific marker of adenocarcinomas of intestinal origin: an immunohistochemical survey of 476 primary and metastatic carcinomas. Am J Surg Pathol. 2003;27(3):303-310.

8. O'Malley DP, Goldstein NS, Banks PM. The recognition and classification of lymphoproliferative disorders of the gut. Hum Pathol. 2014;45(5):899-916.

9. Flodr P, Latalova P, Tichy M, Kubova Z, Papajik T, Svachova M, Vrzalikova K, et al. Diffuse large B-cell lymphoma: the history, current view and new perspectives. Neoplasma. 2014;61(5):491-504.

10. Skinnider BF. Lymphoproliferative disorders of the gastrointestinal tract. Arch Pathol Lab Med. 2018;142(1):4452.

11. Horn H, Schmelter C, Leich E, Salaverria I, Katzenberger T, Ott MM, Kalla J, et al. Follicular lymphoma grade $3 \mathrm{~B}$ is a distinct neoplasm according to cytogenetic and immunohistochemical profiles. Haematologica. 2011;96(9):1327-1334

12. Wen KW, Fakhri B, Menke J, Ruiz-Cordero R, Gill RM, Ohgami RS. Complexities in the diagnosis of large B-cell lymphomas, classic Hodgkin lymphomas and overlapping peripheral T-cell lymphomas simplified: An evidence-based guide. Ann Diagn Pathol. 2020;46:151534.

13. Chuang WY, Chang H, Chang GJ, Wang TH, Chang YS, Wang TH, Yeh CJ, et al. Pleomorphic mantle cell lymphoma morphologically mimicking diffuse large B cell lymphoma: common cyclin D1 negativity and a simple immunohistochemical algorithm to avoid the diagnostic pitfall. Histopathology. 2017;70(6):986-999.

14. Moroch J, Copie-Bergman C, de Leval L, Plonquet A, Martin-Garcia N, Delfau-Larue MH, Molinier-Frenkel $\mathrm{V}$, et al. Follicular peripheral T-cell lymphoma expands the spectrum of classical Hodgkin lymphoma mimics. Am J Surg Pathol. 2012;36(11):1636-1646.

15. Nicolae A, Pittaluga S, Venkataraman G, Vijnovich-Baron A, Xi L, Raffeld M, Jaffe ES. Peripheral T-cell lymphomas of follicular T-helper cell derivation with Hodgkin/ Reed-Sternberg cells of B-cell lineage: both EBV-positive and EBV-negative variants exist. Am J Surg Pathol. 2013;37(6):816-826.

16. Momose H, Jaffe ES, Shin SS, Chen YY, Weiss LM. Chronic lymphocytic leukemia/small lymphocytic lymphoma with Reed-Sternberg-like cells and possible transformation to Hodgkin's disease. Mediation by EpsteinBarr virus. Am J Surg Pathol. 1992;16(9):859-867.

17. Tsimberidou AM, Keating MJ. Richter syndrome: biology, incidence, and therapeutic strategies. Cancer. 2005;103(2):216-228.

18. Xiao W, Chen WW, Sorbara L, Davies-Hill T, Pittaluga S, Raffeld M, Jaffe ES. Hodgkin lymphoma variant of Richter transformation: morphology, Epstein-Barr virus status, clonality, and survival analysis-with comparison to Hodgkin-like lesion. Hum Pathol. 2016;55:108-116.

19. Dojcinov SD, Venkataraman G, Raffeld M, Pittaluga S, Jaffe ES. EBV positive mucocutaneous ulcer-a study of 26 cases associated with various sources of immunosuppression. Am J Surg Pathol. 2010;34(3):405-417.

20. Morita N, Okuse C, Suetani K, Nakano H, Hiraishi T, Ishigooka S, Mori S, et al. A rare case of Epstein-Barr virus-positive mucocutaneous ulcer that developed into an intestinal obstruction: a case report. BMC Gastroenterol. 2020;20(1):9.

21. Moran NR, Webster B, Lee KM, Trotman J, Kwan YL, Napoli J, Leong RW. Epstein Barr virus-positive mucocutaneous ulcer of the colon associated Hodgkin lymphoma in Crohn's disease. World J Gastroenterol. 2015;21(19):6072-6076. 